\title{
LA DISTRIBUCION DE LA RIQUEZA: PARETO DECONSTRUIDO Y GIBRAT RECONSTRUIDO
}

\author{
IGNACIO GONZALEZ GARCIA \\ Departamento de Inteligencia Artificial, ESCUELA TECNICA SUPERIOR DE INGENIEROS \\ INFORMATICOS, UNIVERSIDAD POLITECNICA DE MADRID, ESPAÑA. \\ E-mail: igmigonzalezgarcia@gmail.com \\ ALFONSO MATEOS CABALLERO \\ Departamento de Inteligencia Artificial, ESCUELA TECNICA SUPERIOR DE INGENIEROS \\ INFORMATICOS, UNIVERSIDAD POLITECNICA DE MADRID, ESPAÑA. \\ E-mail:amateos@fi.upm.es
}

\begin{abstract}
RESUMEN
El articulo presenta, utilizando por primera vez los datos completos detallados de una administración tributaria, un análisis de la distribución de la riqueza neta en España, incorporando los datos de los activos y pasivos de todas las personas físicas incluyendo la riqueza derivada de la posesión de acciones en sociedades cotizadas y no cotizadas, con valores actualizados. Se verifica el ajuste de los datos de renta y riqueza a la distribución de Pareto y sus alternativas. Se estudian las limitaciones de los modelos más frecuentemente utilizados en Econofisica. Se muestra la forma en que la distribución de la riqueza puede ser ajustada conforme a la Ley de los números anómalos de Benford para superar la tradicional distinción entre las zonas ajustadas con una curva lognormal y una potencial. Se analizan las regularidades en la distribución de los distintos componentes de la riqueza (patrimonio inmobiliario, activos financieros, etc. ) demostrando su diferente comportamiento. Se propone un método para deconstruir la Ley de Pareto en componentes que permitiría una modelización realista en el ámbito de la Econofísica

Palabras clave: Riqueza, Distribución, Pareto, Gibrat, Econofísica,Benford, activos
\end{abstract}

\section{Wealth's distribution: Pareto deconstructed and gibrat reconstructed}

\begin{abstract}
ABSTRAC
This paper presents, using for the first time the complete detailed data of a tax administration, an analysis of the distribution of net wealth in Spain, incorporating the data of the assets and liabilities of all natural persons including the wealth derived from the possession of shares in listed and unlisted companies, with updated values. The adjustment of income and wealth data to the Pareto distribution and its alternatives is verified. Limitations of the models most frequently used in Econofisica are studied. It is shown how the distribution of wealth can be adjusted according to the Law of Benford anomalous numbers to overcome the traditional distinction between adjusted areas with a lognormal curve and a potential curve. The regularities in the distribution of the different components of wealth (real estate, financial assets, etc.) are analysed and it is explained their different behavior. It is proposed a method to deconstruct the Pareto Law, that would allow realistic modeling in the field of Econophysics
\end{abstract}

Keywords:Wealth, distribution, Pareto, Gibrat, Econophysics, Benford, actives.

Clasificación JEL: C73

Artículo recibido el 16 de febrero de 2019 y aceptado el 24 de mayo de 2019

Artículo disponible en versión electrónica en la página www.revista-eea.net

ISSN 1697-5731 (online) - ISSN 1133-3197 (print) 


\section{LA DISTRIBUCION DE LA RIQUEZA. ANTECEDENTES}

\subsection{La Ley de Pareto}

La distribución de la riqueza ha sido estudiada desde la antigüedad, con un interés algunas veces centrado en la justicia (Rawls, 1971; Sen, 1999) y otras, bien en el conocimiento de su forma, bien en el de sus efectos sobre la economía (Forbes, 2000). Los trabajos de Vilfredo Pareto (1896/1897) atrajeron la atención sobre las regularidades de su distribución, pues mostró, con multitud de ejemplos (Pareto, 1971) que su forma era la potencial, especialmente para la fracción de los ciudadanos más ricos. Quienes han revisado su trabajo lo han hecho en ocasiones (Yakovenko, 2009) buscando una ley universal, mientras que otros lo han criticado (Shirras, 1935) al no encontrar la forma potencial anunciada.

Su difusión impulsó la creación de nuevos modelos tanto de la distribución de la renta como de la riqueza. En 1937, (Champernowe, 1953), utilizó como premisa que la renta de un año depende de la del precedente y de un factor estocástico. Estudió la probabilidad de que la renta de un individuo cambie durante el transcurso de un año desde un rango a otro y demostró que, si la matriz estocástica fuera constante en el tiempo, la distribución tendería hacia un estado de equilibrio, dependiente de la matriz y no de la distribución inicial. Lo ajustó a los datos disponibles en Oxford, que él mismo consideró poco fiables.

A partir de la creación del concepto de Econofísica (Stanley, 2013) confluyeron con los anteriores trabajos los resultados de una corriente de investigación realizada con las herramientas conceptuales propias de la Física. Se han propuesto analogías entre la riqueza y la energía o entre la temperatura y la riqueza media individual. Se han utilizado modelos de colisión para explicar cómo la riqueza se intercambia entre dos agentes económicos por analogía a cómo la energía se intercambia entre dos moléculas, para aplicar luego la teoría cinética de los gases, con la hipótesis de que la riqueza se mantiene constante, como la energía. Otros autores han modelizado el intercambio con un enfoque económico, el de las apuestas, $\mathrm{y}$ alcanzado distribuciones exponenciales para la renta o riqueza, del tipo Boltzmann-Gibbs. Han sido muchas las perspectivas ${ }^{8}$ y muchos los autores (Mandelbrot, 1960; Chakrabarti, 2004; Chakrabrti y otros, 2013; Chatterjee y otros, 2005; Chakrabarti y Chaterjee, 2002). En el enfoque de Chakrabarti cada uno de los agentes mantiene una fracción uniforme y fija de la magnitud considerada, (renta o riqueza). En otros se modeliza la necesaria existencia del ahorro, postulando un factor de propensión que varía entre cero y uno, en cuyo caso la riqueza es siempre positiva.

Otros estudios han conducido a nuevas distribuciones, como la que refleja la maximización de la entropía de Tsallis (Juniper, 2017), que, a su vez, es una generalización de la entropía de Boltzmann Gibbs, o el de Solomon Richmond que generaliza las ecuaciones de Lokta-Volterra de dinámica de poblaciones en presencia de depredadores y presas al ámbito de la economía (Richmond y Solomon, 2002). (Coelho y otros, 2005) han desarrollado un modelo de red familiar que concede gran importancia a la herencia y a las transferencias (dotes y ayudas) que se realizan cuando se crean nuevas familias. Desde una perspectiva más cercana a las Matemáticas, (Mandelbrot, 1960) investigó la distribución de la renta y la aplicabilidad de las distribuciones de Levy-Pareto, que, como la gaussiana, son agregativas y permiten tratar variables como la riqueza por agregación de distintas fuentes sobre distintos colectivos.

Hoy se acepta que la mayoría de las observaciones de renta y riqueza se ajustan razonablemente a distribuciones gamma y lognormales, pero la validez de los modelos no puede ser demostrada por la dificultad en obtener datos detallados, especialmente sobre la riqueza.

El propósito de esta investigación es aportar los datos de la distribución de la riqueza en España, junto con la de sus componentes, para hacer posible la validación del ajuste de los muy diferentes modelos existentes sin sobresimplificaciones y proponer una alternativa sólidamente fundada a la ley propuesta por V. Pareto.

\footnotetext{
${ }^{8}$ Econophysics Forum: www.unifr.ch/econophysics: Consultado 24-05-2019
} 


\section{CONTEXTO DE LA INVESTIGACION}

Hasta el momento ha sido muy difícil disponer de datos suficientemente detallados de la renta y de la riqueza, porque: i) la toma de datos estadísticos está diseñada conforme a los objetivos de cada encuesta y no se adaptan con facilidad a otros propósitos; ii) los datos tributarios tienen una especial protección y no son fácilmente cedidos al mundo académico; iii) porque la riqueza se oculta; iv) porque la riqueza debe ser valorada; $v$ ) porque parte de la riqueza es empresarial y relacionarla con sus titulares es complejo y por último, pero no por ello menos importante, hasta hace pocos años, porque el tratamiento de todos los datos de todas las manifestaciones de la riqueza requiere bastante potencia de cálculo.

V. Pareto estudió los datos disponibles, de ciudades como Arezzo, Parma o Pisa, con el nivel de agregación propio del siglo XIX, cuando no existían recursos informáticos. Otros tratadistas han intentado validar sus conclusiones con estudios históricos. Se citan (Chakrabarty, 2013), estudios sobre sociedades antiguas que, siendo útiles para otros fines, no lo son para éste, pues los conceptos de renta y riqueza están subordinados a los derechos de poder poseer los bienes y transmitirlos. Los resultados en otras culturas (Scheidel y Friesen, 2016), no con comparables con los obtenidos en la sociedad actual. Ya situados en ámbitos homogéneos, los estudios de (Richmond y otros, 2016) aunque más detallados, son todavía insuficientes.

En los últimos años ha despertado el interés académico y social por la desigualdad. Es relevante la obra El capital en el Siglo XXI (Piketty, 2013). Los datos del Banco Mundial (Milanovic, 2018) muestran que la desigualdad entre países creció desde 1980, como se aprecia en The World Inequality Report (2018). (Credit Suisse, 2015) ha publicado series históricas sobre la desigualdad y muchos pensadores han reflexionado sobre ello (Harari, 2018). Saez y Zucman (2016) estudiaron la desigualdad en Estados Unidos, utilizando datos tributarios precediendo un creciente interés académico (Piketty y otros, 2018; Saez, 2018). Filip Novokmet (2018) usó datos de Rusia. En España (Brindusa Anghel y otros 2018), desde el Banco de España, han utilizado datos de la Encuesta Financiera de Familias, para estudiar la concentración de la riqueza en los hogares, midiendo el porcentaje de riqueza poseído por el $1 \%, 5 \%$ y $10 \%$ de aquellos con más riqueza neta, mejorando otros estudios previos. (Luque 2015; Fernández del Cortero, 2015)

Al utilizar datos tributarios se obtiene no solo el beneficio del detalle, sino que, por el hecho de que la Declaración del Impuesto de Sociedades (Formulario 200) permite conocer la participación de los contribuyentes en sociedades no cotizadas, se describen nuevas manifestaciones de la riqueza. Su utilización permite abordar el segundo propósito de esta investigación que es mostrar y justificar las limitaciones de las hipótesis de algunos modelos utilizados en Econofísica, proponiendo alternativas más realistas.

Se ha realizado un estudio en tres fases:

- Cálculo de la renta real neta de cada persona (física o jurídica, pública o privada, residente o no residente, mediante la acumulación de todas las formas de su riqueza, tanto las poseídas directamente, (vivienda, depósitos, etc.) como indirectamente, a través de la participación en sociedades) minorando los activos con los pasivos.

- Cálculo de la distribución de la riqueza neta y de sus componentes, activos, pasivos y sus componentes, y

- Propuesta de una nueva aproximación para la modelización de una ley de distribución de la riqueza.

\section{LA DISTRIBUCION DE LA RIQUEZA EN ESPAÑA}

\subsection{Datos}

Para el conocimiento de la riqueza son insuficientes los datos del Impuesto sobre el Patrimonio. España, desde el año 2011, recuperó esta figura, que obliga a las personas físicas, si su patrimonio es superior a $700.000 €$, después de algunos ajustes. Los datos publicados por la AEAT en su portal referidos a esta declaración tributaria (Modelo 714) cuantifican los "Activos no inmobiliarios" declarados en 442.447 millones de euros ( $\mathrm{M} €$ ), lo que supone un $75,94 \%$ del total, seguido de las 
"Propiedades urbanas y rústicas", $(102.565 \mathrm{M} €)$, que suponen un $17,6 \%$ de un patrimonio total declarado de 582.612 M $€$. Dado que existe un umbral mínimo elevado para la declaración, que la norma legal no establece la obligación de declarar a valor de mercado la participación en empresas no cotizadas y la existencia de reglas especiales de valoración, es evidente que estos datos no reflejan el total del patrimonio neto de los españoles, (que es muy superior a los 582 miles de millones de euros) ni la distribución de la riqueza, ni mucho menos la desigualdad. No pueden ser utilizados, como única base, para estudiar la distribución de la riqueza.

Adicionalmente algunas personas físicas poseen bienes en el extranjero directamente o a través de empresas que a su vez participan en otras empresas, por diversos motivos, entre los que no es descartable el ánimo defraudatorio, como tampoco lo es el intento de quienes quieren blanquear, si es de origen ilícito y ocultar, si es de origen licito, su patrimonio. Para conocer la verdadera distribución de la riqueza todas estas prácticas deben ser desveladas y los velos jurídicos levantados. Describimos a continuación los datos utilizados y los medios empleados para aproximarnos a la realidad desvelada.

\subsection{Colectivo analizado. Censo de personas físicas}

Se han utilizado los datos de la Agencia Estatal de Administración Tributaria (AEAT), relativos a 73.654.776 contribuyentes. Este colectivo, el censo de contribuyentes, está compuesto de: i) personas físicas (60.515.280); ii) personas jurídicas (7.066.844) pertenecientes a 24 categorías (ej.: corporaciones, congregaciones religiosas, fondos de pensiones, sociedades anónimas, compañías de responsabilidad limitada (3.026.153), identificadas por la primera letra de su NIF; iii) personas en situación de baja fiscal por haber cesado su actividad o haber fallecido.

Se observa que el país tiene 46,57 millones de habitantes y más de sesenta millones de personas físicas censadas. Los datos de las personas físicas que exceden a los habitantes corresponden a personas físicas no residentes o personas dadas de baja tributaria por fallecimiento, pero cuyos datos fiscales se conservan por distintas razones, entre ellas por el control de las deudas no prescritas. El número de personas físicas (2015) tratado en la investigación, las existentes en estado de "alta" en el 2015, han sido 59.674.602, número mucho mayor que el de hogares (18.472.800) sobre el que se realiza la Encuesta Financiera de las Familias (EFF) utilizada en otros estudios.

\subsection{Relaciones entre personas físicas}

Puesto que, en ocasiones, determinados bienes son puestos a nombres de parientes o descendientes de anteriores matrimonios o de presentes y pasadas parejas de hecho, se ha utilizado la información sobre las relaciones con el propósito de asignar la totalidad de la riqueza a cada persona física, con la metodología que se explicará más adelante. Aunque desde la perspectiva de la forma de la distribución de la riqueza no tenga mucha importancia, si la tiene desde la perspectiva penal y tributaria.

Los contribuyentes declaran algunas de sus relaciones familiares en la declaración del Impuesto sobre la Renta. Entre ellas se encuentra la relación entre esposos y las existentes con los hijos dependientes. Otras, como las existentes con los hermanos o suegros, si se considera que son necesarias en una investigación, deben ser deducidas. La extensión de las relaciones declaradas se ha realizado de tres modos: i) calculando relaciones como las de hermano o sobrino, algorítmicamente a partir de los datos conocidos; ii) mediante el tratamiento de la serie histórica para encontrar relaciones pasadas, como las de los ex esposos; iii) mediante procesos recursivos, para obtener las relaciones de segundo y tercer grado como las de sobrinos y iv) por minería de datos, para encontrar las existentes entre descendientes de parejas de hecho y los titulares de los bienes, utilizando elementos como la autorización por el titular a menores de edad a hijos de personas con las que se convive. El número de las relaciones declaradas fue de 87.617.949, de ellas 21.843.117 entre esposos, y se han calculado 170.374.613. Se ha creado un grafo con 60.515.280 nodos y 257.992.562 arcos que expresa la totalidad de relaciones encontradas. Las redes familiares declaradas ampliadas del modo descrito forman la red familiar extendida.

\subsection{Riqueza directa e indirecta de una persona física}

La riqueza neta total de una persona física es la suma de la riqueza poseída directamente, de la que forman parte sus propiedades rusticas y urbanas, depósitos bancarios, automóviles, etc., y la poseída 
indirectamente a través de su participación en sociedades, menos sus deudas. Sea la Fig. $1^{9}$. En el lado izquierdo se encuentra una persona física y sus familiares. Se incluyen no solo las relaciones declaradas actuales sino las pasadas y las existentes con familiares como suegros. Es la red familiar extendida. En la derecha una red de empresas unidas por sus participaciones. Por ejemplo, la empresa A, posee un $80 \%$ de la B y un $30 \%$ de la F. La persona considerada y su pareja son socios de las compañías A y F respectivamente y por serlo lo son indirecta y parcialmente de otras, no solo de la Ay la F. Además, ambos poseerán sus bienes personales. La persona A tiene una participación directa en una sociedad off shore a través de A, B y C-

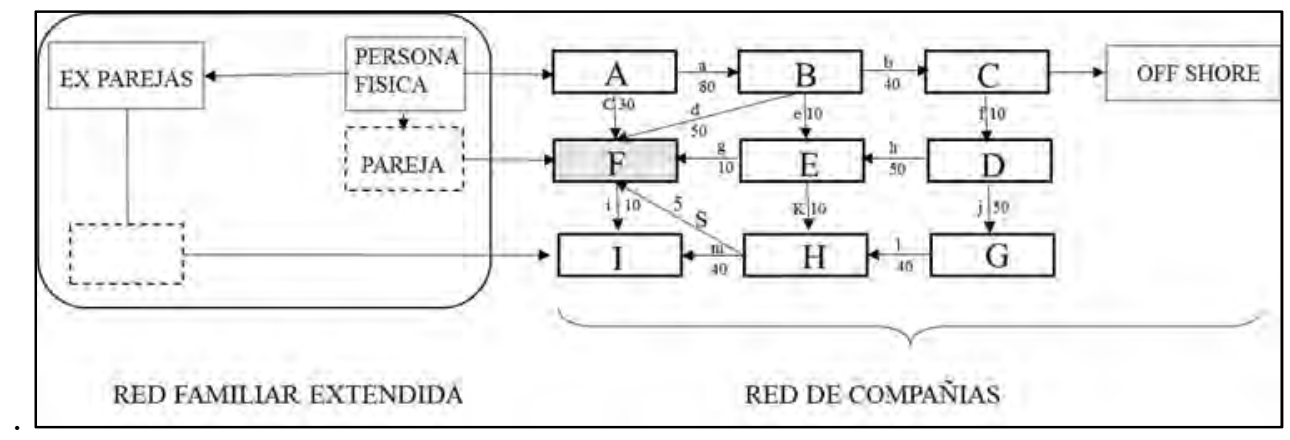

Fig. 1. Relaciones con otras personas físicas y con la red de sociedades

La riqueza poseída a través de participación en empresas cotizadas en Bolsa es conocida por la AEAT mediante la información suministrada por las entidades financieras y se incluye entre los componentes de lo que hemos llamado riqueza directa. En esta investigación se ha reservado el término riqueza indirecta, para la derivada de las participaciones en otras empresas no cotizadas, que son declaradas en el Impuesto de Sociedades, si suponen más de un 5\% del capital. Remarcamos con ello que la distinción entre directamente e indirectamente es convencional y que "indirectamente" describe el modo de posesión de todos los bienes no atribuidos a una persona física y que deben ser obtenidos del grafo de relaciones con otras personas o entidades. En la metodología empleada todo el patrimonio del que se tiene noticia se atribuye primero a una persona física o jurídica y luego, en una segunda fase, a sus verdaderos y últimos propietarios con el procedimiento que, de forma simplificada, se expone a continuación:

(1) La riqueza directa de cada persona física se obtiene sumando los datos de sus componentes: i) inmobiliaria (rustica y urbana), ii) financiera (cuentas y depósitos); iii) otros elementos (fondos de pensiones, pólizas de seguros), etc., con información tomada de los modelos 714, 720, 196, 189, 345. Se han calculado activos y pasivos que incluyen las deudas con la AEAT y las obligaciones con terceras partes para obtener la riqueza neta directa. La riqueza de cada persona jurídica se calcula a partir de los datos del balance utilizando el modelo 200 (Declaración del impuesto de Sociedades).

Se ha utilizado para completar y depurar lo anterior la información imputada por terceros, de la que es muy relevante la suministrada por las entidades financieras. La AEAT dispone de información de cuentas bancarias, suministrada en el modelo 196, que en el año considerado contenía 1.402.306.783 registros, relativos a 79.111.079 contribuyentes con un saldo a favor, a 31 de diciembre de 2017, de 684.424 millones de euros. De ellos 6.182.146 eran personas jurídicas siendo su saldo en el mismo momento $464.782 \mathrm{~m} €$. En todos los casos, para la estimación de los activos y pasivos vinculados, se ha elegido el valor más adecuado considerando: i) el existente en el balance; ii) su valor actualizado, si es el caso, declarado en el modelo 200; iii) el valor derivado de la declaración consolidada del grupo (Modelo 220). Se han ajustado los valores inmobiliarios a valores del mercado tomando el mayor valor entre los siguientes: i) el valor catastral; ii) el utilizado por la Administración en la liquidación de otros impuestos; c) el valor de mercado conocido.

(2) La riqueza indirecta de las personas físicas, derivada de su participación en sociedades no cotizadas, se ha obtenido a través de un procedimiento complejo. Se ha calculado el patrimonio neto de cada sociedad a precios de mercado, actualizado. Hacerlo requiere acumular el verdadero valor de sus

\footnotetext{
${ }^{9}$ Todas las figuras y tablas usan datos de la AEAT y son de elaboración propia.
} 
activos y luego restar sus pasivos. Entre las dificultades se encuentra que los bienes inmuebles muchas veces están declarados a precios de adquisición y que aparecen en los balances amortizados en distinto grado. Además, las empresas pueden formar parte de grupos de empresas. A ello se añade que debe distinguirse en el patrimonio empresarial total, la parte asignada a la actividad empresarial de la que no lo está, lo que es muy importante en pequeños empresarios, en los que su propia vivienda puede estar o no afectada, etc. En otros términos, hay que analizar la contabilidad de cada empresa diferenciando los activos y pasivos vinculados a la actividad empresarial de los que no lo están, para poder realizar el cálculo de las participaciones adecuadamente. Para ello deben considerarse cuentas como las 00188,00153;00160 00223;00238.

Se han considerado también los datos de participaciones en empresas en el extranjero entre ellas 1.507 situadas en paraísos fiscales usando los datos de 24.393 empresas que lo han declarado en el modelo 200, por haber superado los límites establecidos.

Después de realizadas todas las tareas anteriores, para conocer la riqueza indirecta de las personas, se procede a calcular la participación total de cada persona física en cada empresa no cotizada, acumulando la alcanzada por cualquier vía, multiplicando los porcentajes de posesión, por los valores respectivos. Mostramos a continuación el procedimiento.

\subsection{Calculo de la riqueza indirecta. Participaciones}

Al considerar las relaciones entre dos compañías en la Fig. 1, y con ello la existente entre una persona física y una compañía a través de una tercera, observamos la diferencia entre: i) el camino más corto entre ellas; ii) el camino, sea el a-d, a través del cual una empresa, como la (A), posee el máximo de otra (F); iii) el camino más largo. Considerando todos los caminos posibles, se ha realizado con el algoritmo de Pregel, se puede obtener el total porcentaje de participación de una empresa en otra acumulando el porcentaje neto de posesión por cada camino posible. Exponemos el método. Sea la empresa A, de la que la persona física considerada es socio, que mantiene a su vez participaciones en otras empresas, con distintos porcentajes. La compañía B posee acciones en otras: C; F y E. Es el caso que I es parcialmente poseída por $\mathrm{F}(10 \%)$ y que $\mathrm{F}$ y parte de I son poseídas por un pariente (contenido en la relación extendida) del contribuyente, que de hecho es el "beneficial owner".

En algunos casos la relación entre empresa inicial y final implica un solo arco, lo que supone distancia 1. En otros, como el existente entre B y D requiere dos arcos, siendo la distancia 2. La existente a través de a-b-f-j-1-s entre A y F es 6. La participación total se calcula multiplicando las participaciones, mostradas en los arcos. El camino a-d supone una participación del $40 \%(80 \%$ x $50 \%)$ y la participación total se realiza calculando iterativamente, como en los casos a-e-g $(0,08 \%)$ a-b-f-h-g $(0,0016 \%)$ a-b-f-j1-s $(0,00032)$ a-b-f-h-k-s $(0,00008)$ y sumándolos para obtener un $70,0964 \%$

Un total de 2.562.394 contribuyentes tienen al menos una participación en una de estas empresas no cotizadas en Bolsa, por lo que en modo alguno puede despreciarse esta manifestación de la riqueza indirecta. En 266.527 casos la distancia con la persona física es 2, como sucede a A con la empresa B y de ellos en 9.404 casos la persona física las posee al $100 \%$ y en 44.441 casos posee más del $50 \%$. En 54 casos de los 13.240 se posee el $100 \%$ de la empresa oculta a través de cuatro interpuestas.

\section{REGULARIDADES EN LA DISTRIBUCION DE LA RIQUEZA}

\subsection{Leyes de Pareto y de Gibrat}

Se llegó a considerar a la distribución de Pareto la ley más importante encontrada en el ámbito de la economía matemática, pero el transcurso del tiempo ha matizado el optimismo. Otros modelos, (Gibrat, 1931), se ajustaron con más precisión a ciertas zonas de la distribución. Trabajando sobre distribuciones sesgadas, entre ellas las que describen el tamaño de las empresas, Gibrat, quizás basándose en las ideas del astrónomo Kapteyn, afirmó que el efecto observable era resultado de la adición de variables independientes unas de otras y que el resultado se ajustaba a una curva lognormal. El autor, en defensa de su "loi de l'effet proportionnel", asume que el valor de un pequeño cambio en la variable observada es independiente de su magnitud, por lo que su distribución es gaussiana y la variable observada puede ser ajustada por una lognormal. 
Los investigadores han ajustado los datos observados incansablemente a estos modelos desde hace décadas (Baró, 1974; González; 1966; Pena 1965; Alvaredo y Saez, 2009; Richmond, 2006; Milanovic, 2012; Callealta y otros, 1996) por más que algunos no encontraron la distribución postulada. García Docampo (2000), con diversas fuentes de datos, (Encuestas de Presupuestos Familiares del Instituto Nacional de Estadística (INE), Panel de Hogares del INE), en la década de los años 90, encontró índices de Pareto entre 0,8 y 0,9 y ofreció un modelo analítico para el estudio de las desigualdades en la distribución de la riqueza. El que la ley solo haya podido ser verificada empíricamente en algunos casos y en ellos sólo en las rentas más altas, con coeficientes que cambian en cada caso no permite en modo alguno afirmar la existencia de una ley natural y hace muy difícil calibrar y validar modelos. Por eso buscamos ofrecer datos empíricos en cada una de las zonas y para cada uno de los componentes de la curva para alcanzar una precisión superior. El primer paso necesario es definir la curva que se quiere ajustar.

La Ley de Pareto ha sido expresada en la literatura de varias formas. (Mandelbrot, 1960) distingue su forma fuerte que expresamos en (1), donde $\mathrm{m}_{0}$ es un factor de escala, y el valor de $\alpha$ no está determinado. Ambos valores se estiman para cada conjunto de datos analizados.

$$
\Pi(m)=\left\{\begin{array}{cl}
\left(\frac{m}{m_{0}}\right)^{-\alpha} & \text { para } m>m_{0} \\
1 & \text { para } m_{0}<m
\end{array}=\int_{m}^{\infty} P(m) d m\right.
$$

Se debe observar que la función de densidad es proporcional $a m^{-(1+\alpha)}$ Una segunda forma, más débil, afirma simplemente que $\Pi(\mathrm{m})$ tiende a $\mathrm{m} / \mathrm{mo}$ cuando $\mathrm{m}$ tiende a infinito. Simplificando, Pareto afirmó que el número de individuos cuyos ingresos exceden un cierto nivel $\mathrm{m}$ se aproxima bien por la función $C \mathrm{~m}^{-\alpha}$ siendo $\mathrm{C}$ un número real y alfa otro, mayor que cero, debiendo ser ambos ajustados en cada análisis empírico, siendo normal que sea menor que 2.

\subsection{Criterios posibles para el cálculo de la distribución de la riqueza}

Disponiendo de los datos tributarios completos se deben adoptar decisiones:

i) $\quad \mathrm{Si}$ en el modelo se consideran solo a las personas físicas o también las empresas. Se ha considerado la distribución de la riqueza de las personas físicas pues el de las jurídicas se atribuye a aquellas a través de sus participaciones. La de las acciones cotizadas en la riqueza directa y las de las no cotizadas en la indirecta.

ii) Si se considera a todas las personas físicas o solo a las que tienen riqueza. Se ha considerado la de todos aquellos que tienen riqueza sea positiva o negativa, pero no las que no tienen manifestación alguna de riqueza.

iii) Si se utilizan agregados (las familias) o se analiza la distribución de las personas físicas. Muchos estudios utilizan la riqueza de los hogares, cuyo número en el caso español es estimado por el INE. Se trata de una opción razonable y posibilista. Se ha elegido la riqueza individual por tres motivos: el primero es que se dispone de los datos tributarios detallados lo que supone una oportunidad excepcional, el segundo es que, para reflexionar sobre modelos basados en agentes no es lo mismo una opción que otra, no sólo por su tamaño sino porque los miembros de una familia no intercambian riqueza entre sí, en general, mediante el comercio, a diferencia de lo que hacen las familias. Cuando en la modelización se simula el tamaño de las familias, se ha observado que en la curva campanoide $\mathrm{P}(\mathrm{m})$ obtenida, disminuye la altura del pico, para compensar el incremento en su extensión, pues es mayor la riqueza colectiva que la individual y el centro de gravedad de la curva se desplaza hacia la derecha. Parece evidente que la opción que trabaja con agregados, como la familia, es más sensible a las variaciones culturales y a las modificaciones en la edad de emancipación, lo que se aleja del espíritu del investigador en Econofísica. Utilizaremos los datos individuales sin perjuicio de que, si se deseara, pues se conocen las relaciones familiares, la acumulación seria trivial.

iv) Se ha elegido como estimador de la riqueza el valor de los activos de cada persona física. El motivo es importante. El patrimonio neto de todas las personas físicas censadas en 2015 
(59.674.602) fue 4.104.606 millones de $€$ ( 8 veces mayor que el deducido del Impuesto sobre el Patrimonio), con un valor medio de $68.783 €$.

En la tabla 1 se muestran estadísticos descriptivos de varios subconjuntos del anterior y dos variables, activos y patrimonio neto: b.1) personas físicas con patrimonio neto distinto de cero (DIS0); b.2) mayor que cero (MAS0); b.3) con activos mayores que cero y b.4) patrimonios netos negativos. Son la suma total, la media, el valor máximo $(\mathrm{M})$ y mínimo $(\mathrm{m})$, el número de elementos y los deciles de su riqueza neta expresados en euros (2015), para cada colectivo. En la primera columna se ofrece la suma de los patrimonios netos de las personas físicas con activos distintos de cero sea cual sea su patrimonio. Su suma es menor que la segunda pues incluye a quienes tienen patrimonio negativo a diferencia de la segunda donde solo se suma a quienes tienen patrimonio mayor que cero. Son estos 37,025 $\mathrm{M}$ de contribuyentes, 4,5 millones menos que los que tienen algún patrimonio. Todos los que tienen patrimonio tienen algún activo. Las distribuciones de activos y patrimonio neto son similares. Si se caracterizara la riqueza por el patrimonio neto positivo se eliminarían 4.532 .569 casos, que en su mayoría serian personas con vivienda adquirida con un crédito.

Tabla 1

Estadísticos descriptivos de la distribución de la riqueza en España (2015)

\begin{tabular}{|l|r|r|r|r|}
\hline & \multicolumn{1}{|c|}{ DIS 0 } & \multicolumn{1}{c|}{ MAS 0 } & \multicolumn{1}{c|}{ ACT $>\mathbf{0}$} & \multicolumn{1}{c|}{ PN $<\mathbf{0}$} \\
\hline$\Sigma(\mathrm{M} €)$ & 4.104 .606 & 4.228 .499 & 5.221 .882 & -123.893 \\
\hline$\mu$ & 98.769 & 114.205 & 141.009 & -27.335 \\
\hline $\mathrm{M}$ & 4.629 .741 .145 & 4.629 .741 .145 & 4.629 .741 .145 & 0 \\
\hline $\mathrm{m}$ & -164.483 .054 & 0 & -164.483 .054 & -164.483 .054 \\
\hline$\#$ & 41.557 .835 & 37.025 .366 & 37.006 .939 & 4.532 .469 \\
\hline $\mathrm{D} 1$ & -360 & 165 & 171 & -65.663 \\
\hline D2 & 177 & 1.258 & 1.325 & -41.439 \\
\hline $\mathrm{D} 3$ & 1.561 & 5.417 & 6.379 & -27.605 \\
\hline D4 & 7.765 & 18.652 & 27.241 & -18.286 \\
\hline Me & 26.492 & 39.897 & 54.527 & -11.892 \\
\hline D6 & 51.766 & 64.136 & 81.084 & -7.558 \\
\hline D7 & 82.596 & 95.216 & 11.410 & -4.393 \\
\hline D8 & 128.380 & 142.423 & 163.678 & -1.820 \\
\hline D9 & 222.323 & 240.632 & 170.242 & -480 \\
\hline
\end{tabular}

Se deduce: i) la importancia numérica de quienes tienen patrimonio neto negativo (4.532.569 contribuyentes), en la mayoría de los casos con hipotecas inmobiliarias; ii) la existencia de 18.116.767 personas físicas con patrimonio cero, (menores de edad) y de 19.297.595 con valor de activos igual a cero $(52,14 \%$ del total de las personas con activos y un $32,32 \%$ del total). Se debe decidir si se utiliza como variable descriptiva de la riqueza los activos poseídos o el patrimonio neto y si el colectivo es el total, o los que superan un umbral (fue la decisión de Pareto). Se ha entendido que responde más al espíritu del trabajo de Pareto, como al de la Econofísica, trabajar con una expresión de la riqueza cuyos valores siempre son positivos (los valores de la energía que se equipara a la riqueza son positivos) y que tiene una distribución cuyas conclusiones son generalizables. Por ello utilizamos los activos, con lo que se incluye a personas físicas con patrimonio negativo, por ejemplo, asalariados o profesionales con rentas (base imponible media en 2015) de $17.522 €$ y ahorros, que tienen patrimonio negativo explicado por un préstamo hipotecario. Se han eliminado aquellos sin manifestación alguna de riqueza (18.116.767 personas), menores de los que no hay datos.

Es bien conocido que las distribuciones de renta y riqueza tienen formas campanoides y que la altura de su pico ha venido disminuyendo a lo largo del tiempo, desplazándose hacia la derecha a medida que la riqueza y la desigualdad aumentan. En los estudios realizados con datos de U.K. por Chakrabarti, con una muestra del $1 \%$ de los asalariados se encontró el pico en salarios mensuales de 350 libras. Si se 
Tabla 2.

Distribución de la riqueza y sus componentes por tramos

\begin{tabular}{|c|c|c|c|c|c|c|c|c|}
\hline Tramo & $\#$ & P Neto & Activos & Pasivos & Societario & Inmob & Depositos & AA.FF \\
\hline I & 25.848 .476 & 690.527 & 960.691 & 270.163 & 7.836 & 740.756 & 152.095 & 35.935 \\
\hline II & 10.243 .861 & 2.424 .886 & 2.698 .630 & 273.743 & 82.193 & 1.937 .943 & 397.234 & 159.363 \\
\hline III & 378.717 & 819.882 & 991.328 & 171.445 & 222.723 & 400.068 & 67.823 & 141.363 \\
\hline IV & 14.303 .462 & 307.162 & 399.208 & 92.045 & 171.445 & 40.022 & 12.333 & 182.327 \\
\hline $\mathrm{V}$ & 462 & 123.489 & 172.007 & 485.588 & 93.117 & 2.702 & 2.599 & 24.757 \\
\hline Total & 37.006 .939 & 4.365 .868 & 5.221 .882 & 856.014 & 588.196 & 3.121 .467 & 632.064 & 433.957 \\
\hline \multicolumn{9}{|c|}{ COMPOSICION DE LOS ACTIVOS EN PORCENTAJE } \\
\hline $\mathrm{I}$ & & & 100 & 28,12 & 0,82 & 77,11 & 15,83 & 3,74 \\
\hline II & & & 100 & 10,14 & 3,05 & 71,81 & 14,72 & 5,91 \\
\hline III & & & 100 & 17,29 & 22,47 & 40,36 & 6,84 & 14,26 \\
\hline IV & & & 100 & 23,06 & 42,95 & 10,03 & 3,09 & 45,67 \\
\hline $\mathrm{V}$ & & & 100 & 282,31 & 54,14 & 1,57 & 1,51 & 14,39 \\
\hline Total & & & 100 & 16,39 & 11,26 & 59,78 & 12,10 & 8,31 \\
\hline
\end{tabular}

Mientras que, para las rentas bajas su activo inmobiliario, habitualmente financiado con deuda, supone $107 \%$ (740.756/ 690.527) de su patrimonio neto, para los más ricos (Grupo V) el total de sus viviendas y residencias supone el $1,57 \%$. Por el contrario, los más ricos están fuertemente endeudados y aprovechan la ventaja de apalancarse. Lo más importante de apreciar es que el patrimonio en sociedades que no cotizan, el societario, es despreciable en las rentas bajas y supone más de la mitad en las rentas altas. Estos tramos de la sociedad no pueden ser considerados homogéneos desde la perspectiva de la riqueza, como no lo serian desde la perspectiva de rentas porque la distribución de sus componentes de la riqueza no es la misma (no presenta las mismas regularidades).Analizando sus componentes en la Fig. 11 se observa que la distribución del componente patrimonio inmobiliario, se ajusta casi perfectamente a la curva de Pareto para los titulares de patrimonios inmobiliarios entre 10.000 y 20.000.000 de euros. (El gráfico muestra los valores en Ln) Cuando se realiza el proceso con los activos financieros, o con las deudas, no es el caso. Por una parte, las pendientes son distintas, lo que no sería problema, pero si lo es la concavidad hacia las $\mathrm{x}$ en las colas.

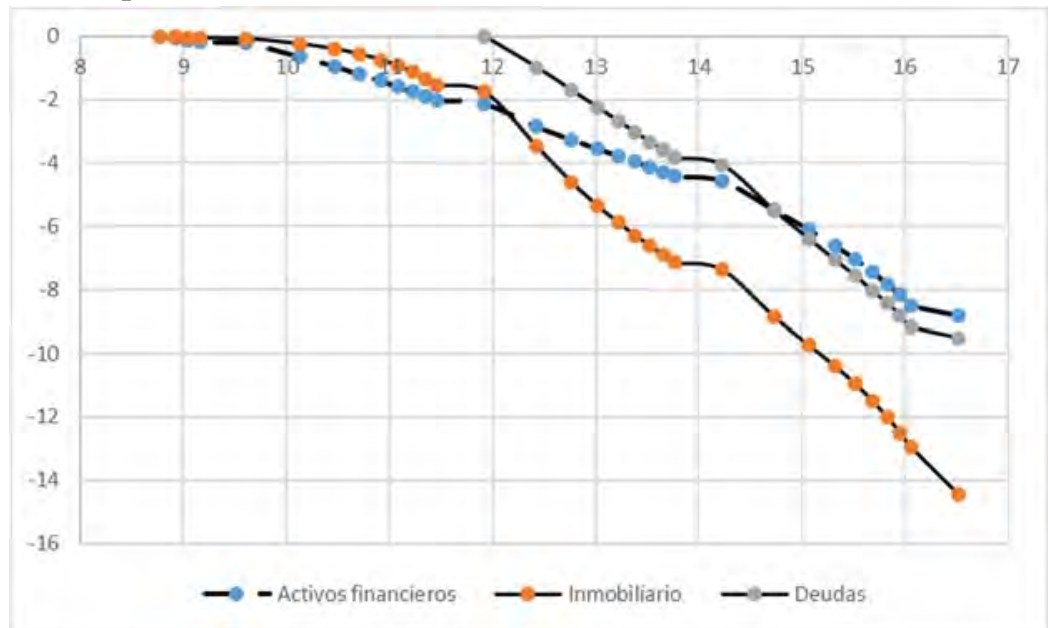

Fig. 11. Distribución de los componentes de la riqueza 
de bombeo hidráulico de excedentes. Las personas obtienen rentas de actividades empresariales e invierten en otros activos además del de la vivienda. El tercer grupo AP, por encima de los $40 \mathrm{M}$ de activos en el caso español, debe modelizarse incluyendo la idea de utilización del endeudamiento para maximizar la riqueza indirecta societaria, en casos excepcionales obtenida y en el general heredada. El sistema total se debe describir por superposición lineal de estos tres colectivos. En el primero el factor decisivo es el consumo, en el segundo la acumulación y en el tercero la distribución.

\section{CONCLUSIONES Y FUTURAS INVESTIGACIONES}

1. Se han ajustado las distribuciones de Pareto de la renta y la riqueza actualizándolos al año 2015 y considerando todos los datos conocidos con las declaraciones tributarias.

2. Se ha propuesto y justificado la necesaria descomposición del colectivo de las personas físicas en tres tipos de poblaciones; trabajadores, acumuladores y administradores, para la realización de modelos.

3. Se ha mostrado que la distribución de la riqueza inmobiliaria, aquella en la que invierte la mayor parte de la población sus ahorros, se distribuye conforme a la Ley de Pareto

4. Se ha mostrado que en la distribución de la riqueza en España no se observan los efectos puntuales del modelo de Coelho.

5. Se ha cuantificado la importancia del efecto de la desigualdad (el $71 \%$ de los tenedores de activos tienen un a riqueza inferior al mínimo paretiano).

6. Se ha mostrado que los estudios de la distribución de la riqueza que no tienen en cuenta los valores actuales de los patrimonios societarios enmascaran la desigualdad en la distribución de la riqueza por omitir el patrimonio poseído indirectamente en sociedades no cotizadas. 2.532.964 (6,26\% del total, los más ricos) participan en estas sociedades, cuya riqueza hasta ahora no considerada en otros estudios triplica en valor, para los más ricos a los activos directamente poseídos.

7. Se ha mostrado la distinta composición de la riqueza de la población en función de los distintos tramos de renta.

8. Se han actualizado los valores obtenidos anteriormente del modelo de Pareto utilizando datos tributarios.

9. Se ha propuesto un modelo de distribución en tres ámbitos, biparamétrico, ajustado al caso español

10. Se ha mostrado la importancia de considerar la normativa y la moneda en que se realizan los cálculos en la modelización, por la presencia de efectos como los descritos en la ley de los números anómalos.

Se pretende, en un próximo trabajo, proponer la expresión de una ley universal de la distribución de la riqueza y de la renta definiendo un modelo que combine los tres submodelos, uno para cada uno de los ámbitos y sus interacciones y proponer un método simple para ajustarlo basado en la distribución de los activos financieros y el patrimonio inmobiliario, por ser datos accesibles que pueden permitir el análisis comparado.

\section{AGRADECIMIENTOS}

Este documento ha sido apoyado por el Ministerio de Economía y Competitividad, proyecto MTM2017-86875-C3-3-R.

\section{REFERENCIAS BIBLIOGRÁFICAS}

ALVAREDO, F. SAEZ, E. (2009), "Income and wealth concentration in Spain from a historical and fiscal perspective", Journal of the European Economic Association. Atn Top Income Global Perspective, Editado por Atkinson, A y Piketty, T: Oxford.

BARO. J. (1974). https://repositori.udl.cat/

BRINDUSA ANGHEL, BASSO, H. BOVER, O. CASADO, J.M. HOSPIDO, L. (2018). "La desigualdad de la renta el consumo y la riqueza en España". Documentos ocasionales del Banco de España, $n^{\circ} 1806$.

CALLEALTA, F.J., CASAS, J.M., NUÑEZ, J. (1996). “Distribución de la Renta per cápita disponible en España: Descripción, Desigualdad y Modelización”. En: Distribución Personal de la Renta en España, Cap. 5, B. Pena (director). Pirámide, Madrid. 
COELHO, R., NEDA ZOLTÁN,RAMASCO, J., SANTOS, A. (2005). "A family-network model for wealth distribution in societies." Physica A 353, 515-528.

CREDIT SUISSE, (2015). Global Wealth Report, 53-ss.

CHAKRABARTI, B., CHAKRABORTI, A, CHAKRABARTY S, CHATERJEE, A. (2013) Econophysics of Income and Wealth Distribution". U.K, Cambridge University Press.

CHAKABRARTI, B.K, CHAHARTEJEE, A., (2004) en "Application of Econophysics, Proc. 2nd. Nikkei Econophys. Symp ,Tokyo, (2004). ed. H. Takayasu, Springer, Tokyo.

CHAMPERNOWE, D.G. (1953). "A model of income distribution", The Economic Journal, 63, 318-351.

CHATERJEE, (2005), "Econophysics of Wealth Distributions". (Ed.: A. Chatterjee, S. y otros), Italia, Milan, Springer-Verlag.

FERNANDEZ DEL COTERO (2015). "Distribución de la riqueza en España: un análisis de la EFF 2011". Electronic resource in Comillas University https://repositorio.comillas.edu/xmlui/bitstream/handle 111531/4640/TFG001341. pdf?sequence=1, accesed, 18, Nov, 2018.

FORBES K. J. (2000). "A reassessment of the relationships between inequality and growth", American Economic Review, 90, 869-887.

GARCIA DOCAMPO, M. (2000). "Medición del análisis de las desigualdades en la distribución de la renta". Empiria. Revista de metodología de las ciencias sociales, 3, 73-99.

GIBRAT, R. (1931). "Les inegalités economiques". Paris: Librairie du Recueil Sirey.

GONZALEZ-QUIJANOS, F., (1966) "La curva de Pareto y la distribución de la riqueza, para rentas superiores a 200.000 ptas". Colegio de Ingenieros de Caminos Canales y Puertos.

JUNIPER, J. (2007). "The Tsallis Distribution and Generalised Entropy: Prospects for Future Research into Decision-Making under Uncertainty", Centre of Full Employment and Equity, The University of Newcastle, Australia.

LANGE, GLENN-MARIE; WODON, QUENTIN; CAREY y KEVIN, W (2018), "The Changing Wealth of Nations, 2018" : Building a Sustainable Future, Washington, DC: World Bank,"

LUQUE, V. (2015). "A propósito de Piketty: evolución de la desigualdad en España”. Papeles de Europa, Vol.28, $86-115$.

MANDELBROT, B.B. (1960). "The Pareto Levy law and the distribution of income". International Economic Review, 1, 79-106.

MILANOVIC BRANCO (2012), "Global Income Inequality by the Numbers: in History and Now", World Bank, Policy Research Working Papers, 6259.

NOAH H., Y. (2018). "21 lessons for the 21st Century", NY, Spiegel \& Grau

NOVOKMET, F, PIKEETY, T, ZUCMAN; G (2017). "From Soviets to Oligarchas; inequality and Poverty in Russia" 1905-2016, July World Wealth and Income Database

PARETO, V,(1971). "Manual of Political Economy". Londres: MacMillan.

PIKETTY, T. (2013). "Capital in the Twenty-First Century”. Harvard University Press.

PIKETTY,T SAEZ,E., y ZUCKMAN G. (2018). "Distributional National Accounts: Methods and Estimates for the United States," Quarterly Journal of Economics, 133, No. 2, May

RAWLS, J. (1971). "A Theory of Justice". Cambridge, M.A.: Harvard University Press.

RICHMOND P., (2006) "A Review of Empirical Studies od Models of Income Distributions in Society" Cap 5 en Econophysics and Sociophysics: Trends and perspectives. En Chakrabarti, B.K. Chackarborti;A. Chaterjee (Eds), Wiley - VCH Verlag GmBH, Weinheim

RICHMOND, P., SOLOMON, S. (2002), "Stable power laws in variable economics; Lotka-Volterra implies Pareto-Zipf" Euro Physics, J. B, 257-261.

RICHMOND, P.HUTZLER;S. COELHO; REPETOWICZ, (2016), "A review of empirical studies and Models of Income Distributions in Society" en el capítulo 5 de "Econophysics and Sociophysics. Trends and Perspectives". Bibas K. Chakrabarti, Anirbam Chakabrorti, Arnab Chaterjee (Eds).

SAEZ, E. Y ZUCMAN, G. (2016), "Wealth Inequality in the United States since 1913: Evidence from Capitalized Income Tax Data," Quarterly Journal of Economics, Vol. 131, No. 2, May

SCHEIDEL, W, FRIESEN, S. (2009), "The size of the economy and the distribution of income in the Roman Empir", The Journal of Roman Studies. 99, 61-91.

SEN, A. (1999). "Poverty and Famines". New Delhi: Oxford University Press.

STANLEY H. E. (2013) "Stanley on Econophysics. (Published in "IIM Kozhikode Society \& Management Review", Sage publication (USA), Vol. 2, Issue 2, (July), 73-78.

SHIRRAS, F. (1935). "The Pareto Law and the Distribution of Income", The Economic Journal, 45; $n^{\circ} 180$, Diciembre,663-681.

YAKOVENKO, V.M, BARKLEY, ROSSER J. (2009). "Colloquium: statistical mechanics of money wealth and income", Review of Modern Physics, 81, 703-1725. 